\title{
Algo de historia del "Bebé Probeta"
}

\author{
Fernando Sánchez Torres*
}

Como bien se sabe, la fertilización in vitro es un método de concepción artificial extracorpórea en el laboratorio. Al primero que se le ocurrió tal procedimiento fue a Paracelso (siglo XVI), quien para ello sembró semen humano y sangre menstrual en estiércol caliente. Su práctica en animales data del siglo XVIII, cuando el abate italiano Lázaro Spallanzani la logró en batracios, siguiendo una técnica científica impecable (4). A finales del siglo pasado, en Inglaterra, Walter Heape comunicó haber logrado con éxito la transferencia de embriones en conejas (2).

Después de Heape muchos otros investigadores reprodujeron las experiencias, con fortuna variable, utilizando distintas clases de animales. Pero el mayor interés fue puesto para lograr el cultivo de embriones en el laboratorio, que permitiría no sólo estudiar el desarrollo embrionario sino también transferirlos de manera selectiva. Uno de los impedimentos era la falta de un medio de cultivo apropiado. Varios fueron ensayados, incluyendo el humor acuoso del ojo. Se debió a John Hammond Jr. haber obtenido el medio que permitió, en 1949, que el óvulo fecundado de ratones se desarrollara in vitro hasta la etapa de blastocisto. M.C. Chang, en 1955, lo logró en conejas (5).

Conviene mencionar que fue G. Pinkus, de la Universidad de Harvard, quien en 1929 cultivó óvulos de coneja fertilizados extracorpóreamente y luego trasplantados en conejas sustitutas, con malos resultados. Utilizando hormonas hipofisiarias para estimular la ovulación, consiguió para su propósito cincuenta y más óvulos. Estas experiencias no las dio a conocer Pincus en su momento. En 1934 se preguntaba si los óvulos de mamíferos podrían, en verdad, desarrollarse normalmente in vitro, sin sufrir modificaciones o desviaciones que hicieran imposible su posterior desarrollo (18). En 1944, John Rock anunciaba con sus investigaciones que sí era posible (19). Recordemos que Pincus y Rock son considerados los "padres de la píldora anticonceptiva".

Volviendo al doctor M.C. Chang, debemos insistir que fue él quien primero lograra la fertilización in vitro de gametos de conejas y su consecutiva transferencia exitosa. El primer nacimiento de crías vivas lo consiguió Chang en 1959 (5).

Los primeros casos de fertilización in vitro y transferencias de embriones en humanos ocurrieron en la década de los 70 así: De Kretzer y colaboradores en Australia, informaron haber transferido un cigoto en 1973 (6). Según el británico Robert Edwards, los datos que respaldaban el informe de De Kretzer no fueron suficientemente sólidos como para tenerlos en cuenta. Douglas Bevis, en 1974, anunció

\footnotetext{
* Profesor Titular, Emérito y Honorario, de la Universidad Nacional de Colombia. Miembro de la Sociedad Colombiana de Historia de la Medicina.
}

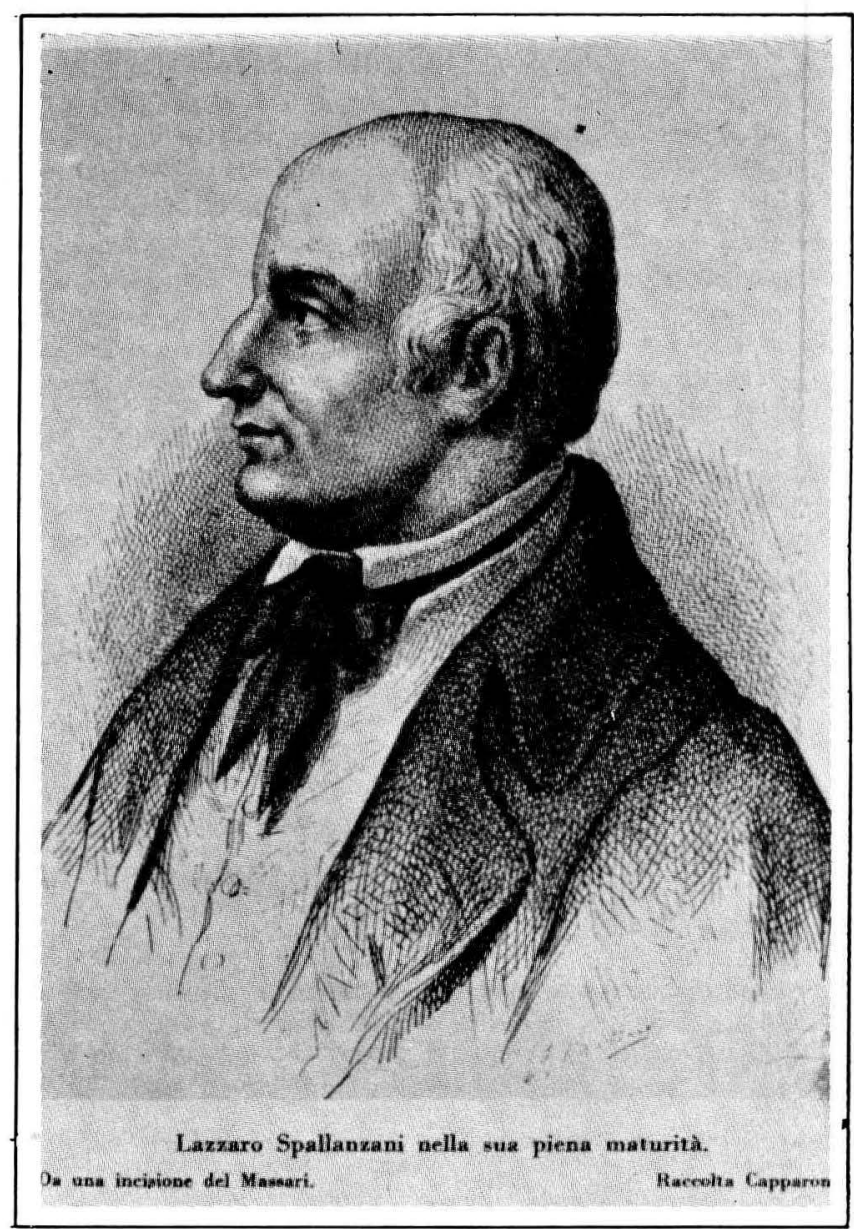

Figura 1. LAZARO SPALLANZANI, científico italiano que lograra en el siglo XVIII la fertilización, en el laboratorio, de un óvulo de rana.

en el British Medical Journal que luego de haber trasplantado embriones había conseguido el nacimiento de varios "bebés probeta" (3). Nadie pudo identificar uno solo de los niños anunciados por Bevis. Esto recuerda al biólogo y genetista italiano Daniel Petrucci, quien en 1959 anunciara, sin demostrarlo, que había logrado el crecimiento in vitro de embriones humanos durante varias semanas. Sus experiencias tampoco tuvieron buen recibo en los medios científicos. Se debe, indudablemente, a los ingleses Robert Edwards y Patrick Steptoe haber alcanzado la meta en 1976 -con un embarazo ectópico- (23) y de nuevo en 1978, esta vez con feliz culminación (24).

El fisiólogo Robert G. Edwards (1926- ), de la Universidad de Cambridge, fue sin duda el cerebro de tan grande 
hazaña. En el campo de la biología se interesó mucho por todo lo relativo a la reproducción humana (8). En 1957, junto con Fowler, en su laboratorio de Edimburgo investigó el fenómeno de la pluriovulación en animales, utilizando gonadotropinas (14); en 1965 comunicó haber logrado la maduración in vitro de óvulos de mamíferos, incluyendo humanos (7); en 1973 consiguió la fertilización in vitro de óvulos de vaca. Entonces decidió traspasar esta experiencia también a la especie humana. Para ello se asoció con un inquieto ginecobstetra que trabajaba en la pequeña ciudad de Oldham: Patrick Steptoe (1912-1989), antiguo marino de la Armada Real y prisionero durante la Segunda Guerra Mundial. Su carrera de investigador comenzó tarde, a la edad de 39 años. Uno de sus primeros trabajos fue un corto libro titulado Laparoscopia en Ginecología, publicado en 1967, que ha sido considerado como el vehículo introductor de dicho procedimiento exploratorio en los países de lengua inglesa. Precisamente el que Steptoe fuera un experto en el manejo del laparoscopio condujo a Edwards a buscarlo como socio. Más adelante veremos qué buen socio fue.

En concepto de Edwards, lo más relevante era poder conocer el momento adecuado de la maduración del oocito humano in vitro e in vivo, como también de otros mamíferos, pues así se podría calcular el momento de la ovulación y practicar a tiempo la laparoscopia para rescatar el óvulo. Con el propósito de recoger dos o tres oocitos de cada mujer sometida al procedimiento de la fertilización in vitro, acordaron utilizar hormona gonadotrópica en dosis bajas, ya que el clomifene no producía los suficientes folículos. Después de múltiples observaciones llegaron a la conclusión de que el momento oportuno de la laparoscopia para captar los óvulos correspondía a las 36 horas después de aplicada la gonadotropina, que es cuando los cromosomas de los oocitos han alcanzado en su división la etapa de metafase (22). Más o menos dos horas después el oocito estaba dispuesto a recibir el espermatozoide. El gameto macho debía estar también maduro, vale decir, capacitado para cumplir su cometido. Edwards y Steptoe tuvieron éxito lavando apenas los espermatozoides eyaculados y llevándolos a la fertilización. Se creía que la fertilización in vitro sólo era posible si se utilizaban espermatozoides que habían alcanzado la cavidad uterina o las trompas de Falopio, ya que únicamente así, según C.R. Austin, el gameto macho alcanzaba su capacitación (1). Como era natural, las primeras publicaciones de Steptoe y Edwards que anunciaban el éxito de la fertilización in vitro fueron recibidas con asombro e incredulidad por cuanto ellos habían omitido la etapa de capacitación del espermatozoide. Comentando este hecho dijo Edwards: "No es necesario decirlo. Patrick estaba encantado con su ingreso exitoso al campo investigativo de la embriología humana" (9).

Vino luego el problema de conocer el medio adecuado en que debía mantenerse el huevo humano fecundado y el consiguiente embrión. El biólogo Barry Bavister producía un buen medio para fertilizar huevos de hámster in vitro; ellos lo usaron con buenos resultados, tomando las medidas necesarias para evitar que se infectara el cultivo, que se evaporara o que perdiera el $\mathrm{pH}$ adecuado. Así pudieron ver, con la natural emoción, cómo los embriones crecían de 2 células a 4 , y de 4 a 8 . El núcleo y los cromosomas de algunos embriones fueron examinados. No obstante que to- dos parecían estar bien, no trasplantaron ninguno hasta que muchos blastocistos fueran mejor examinados.

Después de dos años de febril investigación, Steptoe y Edwards creyeron conveniente trasladarse de Oldham a Cambridge y abrir un laboratorio allí, pero el Consejo de Investigación Médica se negó a apoyarlos, aduciendo que la laparoscopia era peligrosa y que era mejor continuar los estudios en monos rhesus antes de hacerlo en humanos. Era un duro golpe para ellos, pues Steptoe sabía que había practicado miles de laparoscopias sin ningún problema y Edwards sabía que el estudio en monos no tenía objeto ya que la ovulación, la blastulación y la implantación eran muy diferentes en la especie humana. Por eso decidieron continuar trabajando por su cuenta. El siguiente paso fue la transferencia de embriones a sus mismas madres, es decir, a aquellas que habían suministrado los óvulos. Todas las transferencias que llevaron a cabo entre 1971 y 1975 fracasaron, sin explicarse la razón, pues la estimulación folicular era excelente, los embriones parecían espléndidos y las siembras habían sido efectuadas con 4, 8 y hasta 16 células. Dice Edwards que Steptoe, frente a este fracaso repetido, dio muestras de la fortaleza de su carácter. Rehusó darse por vencido, porque deseaba ayudar a los pacientes que habían confiado en él. Tiempo después creyeron que la razón de su fracaso residía en una falta de soporte progestacional, dado que la gonadotropina coriónica reducía la fase luteínica a siete u ocho días después de la aspiración folicular. Entonces suministraron progesterona de sustitución, mientras la placenta era capaz de sostener el embarazo. Nuevamente el fracaso: la progesterona de depósito que utilizaron no resolvía el problema y las pacientes abortaban a corto plazo.

Los primeros años del 70 fueron para ellos difíciles. No sólo tenían que sostener el programa en Oldham, sino que además era permanente el asedio de la prensa y de los medios científicos. Las pacientes entraban ilusionadas a la clínica y salían defraudadas. Entonces se trasladaron a un pequeño hospital en Kershaw, donde instalaron el primer laboratorio en el mundo de fertilización in vitro. Allí continuaron trabajando, lejos de las presiones de un gran hospital.

Pero dejemos que sea Edwards quien continúe la narración de este apasionante capítulo:

"Entonces ocurrió el mayor avance. Una paciente con un embrión transferido presentaba clínicamente un embarazo. Infortunadamente desembocó en una gestación ectópica (...). Este embarazo fue para nosotros el mayor progreso, no obstante que muy pocos de nuestros colegas creyeron que de verdad había ocurrido luego de una fertilización in vitro. Este acontecimiento fue decisivo puesto que ahora sabíamos que el crecimiento de los embriones in vitro y el método de transferencia eran correctos, como también que el soporte hormonal a los tejidos de la trompa era satisfactorio y que los seres humanos podían tolerar la superovulación y sostener un embarazo. Era perfectamente posible que el embarazo ectópico hubiera sido un insólito golpe de azar. Pudo haber fallado el implante, lo que llevó al embrión a emigrar a la trompa. Esto nos condujo a cuestionar el uso de la progesterona de depósito como soporte para el útero, y desde entonces se abrieron otras opciones que debíamos ensayar con el fin de conseguir que el endometrio nos permitiera lograr nuestro primer bebé. Irónico el que hoy, muchos de aquellos que dudaron que este embarazo fuera lo- 
grado in vitro, tengan también sus propios embarazos ectópicos siguiendo el mismo procedimiento (...). Finalmente, decidimos monitorizar, mediante dosificaciones hormonales seriadas, el ciclo menstrual espontáneo en un intento de captar un oocito maduro único, fertilizarlo y transferirlo en un ciclo en el cual el soporte de progesterona natural pudiera ser adecuado.

“Los años 1977 y 1978 fueron fascinantes: sin apoyo financiero, nosotros fuimos estableciendo un programa de congelación de óvulos y embriones, y un modesto laboratorio de endocrinòlogía para monitorizar la $\mathrm{LH}$ urinaria en muestras recolectadas cada tres horas durante el día. El equipo Hi-Gonavis (Mochida Pharmaceuticals) nos permitió detectar las variaciones del ciclo natural y predecir oportunamente el momento para captar los óvulos maduros. Para sorpresa nuestra, la gran mayoría de los 'picos' de LH comienzan cerca de las tres de la madrugada, observación esta fundamental en endocrinología humana. Nuestra segunda paciente del ciclo menstrual natural, espontáneo, fue Lesley Brown quien, como el mundo sabe, logró un embarázo exitoso, así que nosotros abandonamos los otros tratamientos. El retiro de Patrick, por edad, del Servicio Nacional de Salud ocurrió durante el embarazo de Lesley Brown, así que llegó en un momento crítico.

"Patrick Steptoe mostró su temple nuevamente durante el embarazo de Lesley Brown. El embarazo llegó a conocimiento de la prensa, y el gran número de periodistas le ocasionó a ella demasiado estrés, tanto que tuvo que dejar su hogar, pues para la salud del feto era amenazante. Patrick organizó su cuidado en un lugar seguro durante los últimos meses de embarazo, llevándola finalmente al Hospital General de Oldham. Todas las medidas fueron tomadas allí, iy transmitidas a la prensa por un espía infiltrado en el hospital! El feto fue monitorizado cuidadosamente con ecografías y su crecimiento era normal; Patrick llevó a cabo el parto mediante operación cesárea, en total secreto y con éxito completo. Entonces corrió la versión de que había una bomba en el hospital y que era necesario evacuar la sala de maternidad, versión puesta en circulación por alguien que deseaba conseguir una fotografía de Lesley a cualquier precio. Sobra decirlo, la fotografía no fue tomada.

"El nacimiento de Louise obviamente fue el punto más alto en nuestra carrera de la fertilización in vitro. Pero si no hubiera sido ese el punto más alto, para nosotros significó una serie amplia de nuevos descubrimientos: la recolección de óvulos maduros, la fertilización in vitro, el maravilloso crecimiento de embriones hasta el día 5 y hasta el día 9 a través de incubación (una de las más hermosas visiones) y el embarazo ectópico en el segundo trimestre, con un feto desarrollado completamente normal (...)" (9).

El anterior relato, un poco extenso, lo he transcrito por considerar que él recoge, por mano de uno de sus actores, las intimidades de uno de los hechos más trascendentales en toda la historia de la reproducción humana: el "bebé probeta", o "bebé del siglo", como lo llamó la prensa internacional (27), o el "bebé espectáculo", como lo denominó el investigador francés Jacques Testart, autor en 1982 de la primera fertilización in vitro y transferencia de embrión, exitosas, llevadas a cabo en Francia (25).

El nacimiento de Louisa Brown ocurrió el 25 de julio de 1978, fecha importante en los anales de la historia de la

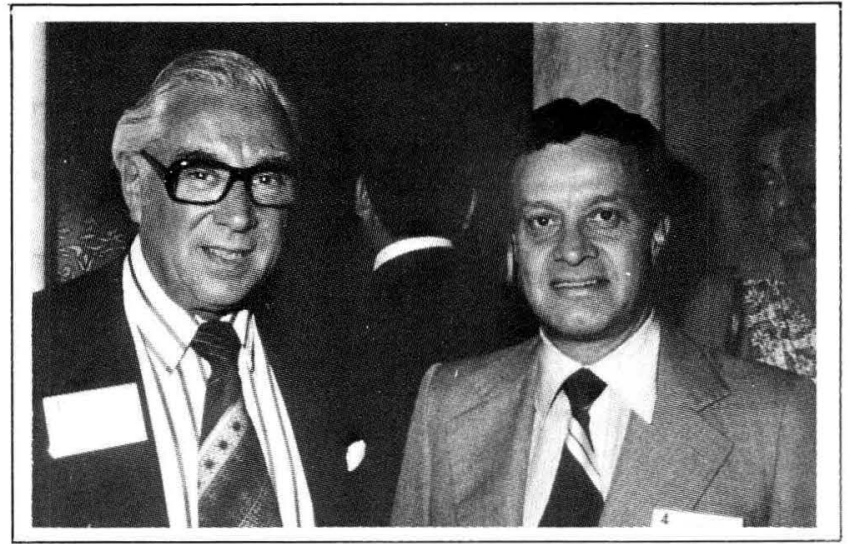

Figura 2. PATRICK STEPTOE, uno de los padres del bebé probeta, con el autor de esta reseña histórica (1979).

medicina. Lesley Brown, su madre, era una mujer de 30 años, residenciada en Bristol. Desde hacía nueve años, junto con su esposo Gilbert John, anhelaban fervientemente un embarazo.

Algunos detalles más, referidos por Patrick Steptoe al autor de esta reseña (21). De 32 intentos para implantar in útero óvulos fecundados in vitro, solamente en cuatro lo lograron. Algo sorprendente: esos cuatro embarazos ocurrieron después de haber hecho la siembra entre las 10 y las 12 de la noche. ¿Coincidencia? Quizás. Recordemos que líneas atrás Edwards llamó la atención acerca de que la gran mayoría de los "picos" de LH comenzaban a observarse hacia las tres de la madrugada. Para Steptoe el ritmo circadiano pudo jugar un papel importante en el resultado exitoso. Dos de las mencionadas gestaciones se frustraron precozmente, una a las 11 semanas, comprobándose daño cromosómico en el embrión (triploidia); la otra a las 20 semanas, sin que la autopsia demostrara malformación alguna. De las dos restantes una correspondió a Louisa Brown y la otra a un varón, Alistair. Al divulgarse que en la experiencia de Steptoe y Edwards se había presentado un caso de malformación cromosómica, surgió el temor de que con la fertilización extracorpórea pudieran obtenerse niños anormales. Al respecto Steptoe expresó: "Cerca del $50 \%$ de los óvulos que se fertilizan de manera natural no llegan a la fase de implantación porque en su intimidad hay algo equivocado, anormal. La naturaleza frustra la fertilización cuando el óvulo o el espermatozoide presentan defectos cromosómicos. Lo mismo puede aplicarse a la fertilización in vitro. Aún más, la incidencia de anormalidades fetales puede ser más baja que la observada en la fertilización natural ya que se pueden desechar aquellos embriones que no crecen adecuadamente en la fase de preimplantación".

En la década de los sesenta la aparición de la píldora anticonceptiva se constituyó en un verdadero "boom" que retumbó en todo el mundo. Finalizando la década de los setenta, la aparición de Louisa Brown, el "bebé probeta", tuvo el mismo efecto. Una década después, pasada esa conmoción, cabe aquí la pregunta: ¿Fue la simple curiosidad lo que llevó a los científicos a utilizar la tecnología para alcanzar tan grande hazaña? Lo que inició Heape en los años finiseculares y que tenía como objetivo la reproducción 
animal artificial con fines mercantiles, se hizo en la especie humana, no hay duda, con el objeto de beneficiar al núcleo social primario: la familia. Creo que razones tales como la frecuente ocurrencia de la esterilidad femenina por daño de las trompas de Falopio, el relativo éxito de su reparación quirúrgica y la dificultad en los países occidentales -particularmente en los llamados "opulentos"- de lograr una adopción a causa de la extinción de los niños expósitos, fenómeno explicado a su vez por la utilización de métodos anticonceptivos, por la liberación del aborto y por el creciente apoyo a la madre soltera, fueron, digo, las razones para que el empecinamiento de Steptoe y Edwards lograra tan insólita hazaña: la de concebir hijos extracorpóreamente. Por supuesto que tal adelanto, considerado un imposible veinte años atrás, ha traído consigo muchos interrogantes y muchos conflictos de carácter ético. Conocido el nacimiento de Louisa Brown, el doctor John Marshall, del condado de Los Angeles, al ser interrogado por un periodista de la revista Time, expresó: "El potencial de esta desgracia es ilimitado" (27).

A la muerte de Patrick Steptoe, ocurrida diez años después de haber dado a luz al "bebé probeta", más de 1.000 niños habían nacido por ese método. Por tratarse de un procedimiento costoso, comenzó a ponerse en práctica en laboratorios privados en algunas naciones de Europa y de América. En Francia, segundo país del mundo donde se llevó a cabo, su realizador, e! biólogo Jacques Testart, jefe del Instituto Nacional de Investigación Médica, recogió velas y se declaró en desacuerdo con el método, por razones morales. En su libro L'oeuf transparent, aparecido en 1986, advierte que se ha llegado a un momento en que el científico debe autolimitarse, ya que la investigación no es neutra y porque es su deber pensar en las implicaciones éticas que conlleva cada descubrimiento antes de lograrlo, y no después (26).

En Colombia, como en otros países, ha habido controversia acerca de la prioridad nacional. El diario El Espectador, de Bogotá, sorprendió a los círculos científicos y a la opinión toda con la noticia, el domingo 14 de octubre de 1984, de que en Cali, en la Clínica de Occidente, había nacido el primer bebé probeta como resultado de un proyecto de fertilización in vitro adelantado por el Instituto de Reproduc- ción Humana y Profamilia, dirigido por el doctor Gary Ventolini (12). Y digo que fue sorpresa por cuanto se ignoraba en los círculos científicos que en Colombia se estuviera llevando a cabo la fertilización in vitro. De ahí que la Sociedad Vallecaucana de Obstetricia y Ginecología convocara a un foro para dilucidar el asunto, llegándose a la conclusión de que "(...) no hay evidencia científica que asegure que el caso presentado en Cali como «El primer bebé probeta de Latinoamérica» sea evidentemente una fertilización in vitro y una transferencia de embrión" (20). La circunstancia de que el equipo científico dirigido por el doctor Ventolini no siguiera trabajando en ese campo, echa todavía más sombras a la veracidad de la noticia periodística.

En Bogotá ocurrió algo similar, en cuanto a la manera de dar a conocer el hecho. La prensa nacional $(10,13)$ y la internacional (11) recogieron el sábado 12 de enero de 1985. la noticia de que en la noche anterior en la Clínica del Country había culminado exitosamente, y mediante operación cesárea, la gestación de Piedad Romero -arquitecta bogotana-luego de una fertilización in vitro y transferencia de embrión. La señora Romero había sufrido anteriormente extirpación quirúrgica de las trompas de Falopio.Los autores de esta experiencia fueron el doctor Elkin Lucena Quevedo y su equipo de colaboradores, quienes de tiempo atrás estaban dedicados al estudio y tratamiento de la infertilidad. Extraña el hecho de que los trabajos del doctor Lucena no hubieran sido divulgados a través de publicaciones científicas. De ahí que para respaldar el hecho histórico no se encuentren fuentes distintas al registro periodístico. De todas maneras, la circunstancia de que el Centro Colombiano de Fertilidad y Esterilidad (Cecolfes), dirigido por el doctor Lucena, sea tenido como uno de los más importantes en su género en América Latina -como que recibe especialistas de otros países para adiestrarlos en el procedimiento del bebé probeta (15) - pone piso firme a la ocurrencia del nacimiento de Diana Carolina, la niña engendrada por método artificial. Este caso ha sido considerado como el primer nacimiento en América Latina luego de fertilización in vitro. Así lo ha declarado el doctor Roberto Nicholson, presidente de la Federación Latinoamericana de Sociedades de Esterilidad y Fertilidad $(16,17)$.

\section{BIBLIOGRAFIA}

1. AUSTIN, C.R. Observations of the penetration of the sperm into the mammalian egg Aust $J$ Sci Res 1951; 134: 581

2. BETTERIDGE, K.J. An historical look at embryo transfer $J$ Reprod Fert 1981: 62: 1 .

3. BEVIS, D.C.A. Embryo transplants Br Med J 1974; 3: 238.

4. CAPPARONI, P. Spallanzani. Unione Tipografico, Torino, 1941.

5. CHANG, M.C. Fertilization of rabbit ova in vitro. Nature 1959 ; 184: 406

6. DE KRETZER, D.; DENNIS, P.; HUDSON, B. et al. Transfer of human zygote. Lancet 1973; 2: 728.

7. EDWARDS, R.G. Maduration in vitro of mouse, sheep, cow, pig, rhesus monkey and human ovarian oocytes. Nature 1965; 208: 349.

8. EDWARDS, R.G. Studies on human conception. Am J Obstet Gynecol 1973; 117: 587

9. EDWARDS, R.G. Tribute to Patrick Steptoe: beginnings of laparoscopy. Human Reprod 1989; 4: 1 (suppl.).

10. EL COLOMBIANO. Bebé probeta de Bogotá, primero de Latinoamérica, 1985; enero 12, p. 3A, Medellín.

11. EL COMERCIO. Primer bebé probeta nace en Colombia, 1985; enero 12, p. 1, Lima.
12. EL ESPECTADOR. En Cali, primera niña probeta, 1984; octubre 14 , p. 12A, Bogotá.

13. EL TIEMPO. Nació bebé probeta colombiano, 1985; enero 12, p. 1, Bogotá.

14. FOWLER, R.E.; EDWARDS, R.G. Induction of superovulation and pregnancy in mature mice by gonadotrophins. I Endocrinol 1957; 15: 374 .

15. LUCENA, E. Comunicación personal. Bogotá, febrero, 1991.

16. NICHOLSON, R.F. Carta personal al doctor Lucena, Buenos Aires, agosto 23, 1990.

17. NICHOLSON, R.F.; CHILLIK, C.F.; LUCENA, E. et al. Fertilización in vitro en Latinoamérica. Rev Latinoam Esteril Fertil 1990; 4: 1 .

18. PINCUS, G.; ENZMANN, E.V. Can mammalian eggs undergo normal development in vitro? Proc Nat Acad Sci 1934; 20: 121.

19. ROCK, J.; MENKIN, M.F. In vitro fertilization and cleavage of human ovarian eggs. Science 11944; 100: 105.

20. SOCIEDAD VALLECAUCANA DE OBSTETRICIA Y GINECOLOGIA. Carta al presidente del Tribunal de Etica Médica del Valle del Cauca, Cali, febrero 5, 1985. 
21. STEPTOE, P. Comunicación personal. Seúl, Korea, mayo 9, 1979.

22. STEPTOE, P.C.; EDWARDS, R.G. Laparoscopic recovery of preovulatory human oocytes after priming of ovaries with gonadotrophins. Lancet 1970; 1: 683.

23. STEPTOE, P.C.; EDWARDS, R.C. Reimplantation of a human embryo with subsequent tubal pregnancy. Lancet 1976; 2: 880.
24. STEPTOE, P.C.; EDWARDS, R.G. Birth after reimplantation of a human embryo. Lancet 1978; 2: 366.

25. TESTART, J. De l'éprouvette au bébé spectacle. Editions Complexe. Bruxellas, 1984.

26. TESTART, J. L'oeuf transparent. Flammarion, Francia, 1986.

27. TIME. The firts test-tube baby, 1978; july 31 , p. 28

\title{
INFORME CONGRESOS
}

XVIII CONGRESO COLOMBIANO DE OBSTETRICIA Y GINECOLOGIA. Diciembre 4-5-6 y 7 HoteI El Prado. Barranquilla. Informes: FECOLSOG. Tel.: 2681485, Bogotá. Tels.: 359604 - 560546, Barranquilla

II $^{\text {nd }}$ WORLD CONGRESS FOR INFECTIOUS DISEASES IN OBSTETRICS AND GYNECOLOGY. September 7th, - 14 th, 1991 BANGKOK, THAILAND. Abstract forms, Registration forms and Information: The Secretary, $\mathrm{II}^{\text {nd }}$ World Congress for Infectious Diseases in OB/GYN, P.O.B. 702027,8000 Munich 70, FRG. FAX 0049/89/7095/8884.

XIII CONGRESO MUNDIAL DE OBSTETRICIA Y GINECOLOGIA, Figo 1991, del 15 al 20 de septiembre de 1991, Singapur. Informes e inscripciones: Dr. P.C. WONG, Secretario General del Comité Organizador, O. \& G., Society of Singapure. C/O/Dept. of Obstetrics \& Gynaecology. National University Hospital. Lower Kent Ridge Road. Singapore 0511. Republic of Singapore. Tel. (65) 3382154/3382314. Telefax: (65) 3382351. Télex: UNISPO RS 33943.

\section{NOTICIAS FIGO}

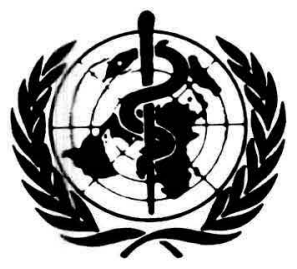

\author{
FOCUS: MORTALIDAD MATERNA \\ UNA TRAGEDIA SILENCIOSA \\ (Traducido de Figo 31 (3): marzo 1990)
}

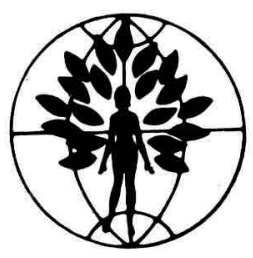

Pocas mujeres del hemisferio occidental se imaginan que ellas morirán durante el proceso del parto. Pero para la mayor parte de ellas, que viven en los países en vías de desarrollo, las perspectivas son demasiado reales.

La Organización Mundial de la Salud (OMS) estima que cerca de 500.000 mujeres mueren cada año como resultado del embarazo, más del $99 \%$ de ellas en países del tercer mundo. Las tasas de mortalidad materna, alrededor de 500 por 100.000 nacidos vivos son citadas para el mundo en vías de desarrollo comparado con 10 a 30 para los países desarrollados.

Esta trágica pérdida de vidas es producto principalmente de tres causas: servicios de salud para mujeres embarazadas totalmente inadecuados, falta de disponibilidad de servicios de planificación familiar y a los efectos de la pobreza crítica. Esta última exacerbada por la diseminación sexual, socava la salud de la mujer desde su infancia en adelante y predispone a un patrón reproductivo particularmente riesgoso con embarazos ocurriendo muy temprano o muy tarde en la vida reproductiva así como con intervalos mínimos entre cada uno de los embarazos.

Los asesinos principales de la mujer en su maternidad son los siguientes: hemorragia, infección, toxemia, distocia de trabajo de parto y las complicaciones derivadas del aborto provocado. La anemia y otras enfermedades crónicas están frecuentemente presentes como factores contribuyentes. Se puede hacer mucho para prevenir esas muertes aun cuando existan recursos limitados. Pero ello requiere audacia y nuevas ideas en las políticas de los programas dirigidos hacia la mayoría de las mujeres que viven actualmente y que no reciben los beneficios de la medicina moderna.

La OMS ha redactado algunas directrices a seguir en este tema. Ellas son:

a) Creación de servicios eficaces que lleguen a los niveles más periféricos del sistema de la salud, y b) Tener un personal entrenado capaz de realizar todos los procedimientos necesarios.Las comadronas y las obstetrices tradicionales pueden ser capacitadas para la atención de partos bajo adecuadas condiciones sanitarias y de esa manera reducir el riesgo de infección. La incidencia de toxemia puede ser disminuida al proveerle a esas gestantes un buen control prenatal a nivel de la comunidad.

La hemorragia y las distocias del trabajo de parto, sin embargo, casi siempre requieren facilidades quirúrgicas y personal profesional especializado y éstas a su vez ameritan cierta infraestructura hospitalaria. Ciertamente redes de pequeños hospitales de distrito o centros de salud con camas de hospitalización son esenciales para ofrecer servicios de emergencia.

La planificación familiar tiene un importantísimo papel para reducir el número de embarazos de alto riesgo: espaciar las gestaciones y prevenir aquellos embarazos indeseados que puedan terminar en aborto ilegal. Estos servicios deben expandirse tanto como sea posible para la mejor disponibilidad de las usuarias.

Por un bajo costo por persona dedicado a la salud, la mortalidad materna puede ser reducida a la mitad en menos de una década. Debe ser una meta a alcanzar dentro de una voluntad política. Los profesionales de la salud deben estimular dentro de sus actividades esa voluntad tan plausible como necesaria. 